\title{
Using Molecular Dynamics Simulations to Understand Electron Beam Interactions with Macromolecules in Liquid-phase Transmission Electron Microscopy
}

\author{
John Smith ${ }^{1}$, Chang Liu $^{1}$ and Qian Chen $^{2}$ \\ ${ }^{1}$ University of Illinois at Urbana-Champaign, United States, ${ }^{2}$ University of Illinois at Urbana-Champaign, \\ Urbana, Illinois, United States
}

Through direct nanoscopic imaging in solution, liquid-phase transmission electron microscopy (TEM) has the potential to provide fundamentally new understanding of macromolecules, whose structure and functional dynamics are defined by a surrounding solvent [1]. For example, synthetic polymers can adopt either a collapsed or random coil-like configuration depending on the balance of segment-segment and segment-solvent attractions. Proteins, the polymers of nature that provide molecular functionality in living cells, adopt a three-dimensional (3D) conformation determined by solvation and the solvent-mediated synergy of electrostatic, hydrophobic, and van der Waals interactions. As a result, great efforts have been made to use liquid-phase TEM to image macromolecular systems, such as phase-separating block copolymers [2], hydrated viruses [3], and structural proteins [4]. However, interpretation of the obtained liquid-phase TEM movies or images is often difficult because macromolecules are in general highly sensitive to electron beam damage. Shape transformations observed under liquid-phase TEM could thus be attributed either to generic configurational fluctuations governed by thermal agitation in a solvent or simply beam-induced structural alterations. Moreover, the low TEM contrast of macromolecules, combined with the need for low electron dose rates to minimize beam damage, leads to TEM movies or images of low signal-to-noise ratio. Detailed analysis and assignment of the intensity profiles of macromolecules -where specific molecular-level features lie - has thus been limited in fidelity.

Here we demonstrate a prototype workflow in which molecular dynamics (MD) simulations are used both to aid in the evaluation of beam artifacts and to infer molecular-level information from otherwise difficultto-interpret liquid-phase TEM movies of macromolecules. In particular, we generate "hypotheses" for distinct atomic arrangements of macromolecules based on all-atom MD simulations with explicit solvent molecules. Although covering limited time scales (usually below $\mu \mathrm{s}$ ), MD simulations can generate a large library of macromolecular structures "expected" at equilibrium. These coordinates are then combined with electron optical theory (considering factors such as noise, defocus and the effects of the solvent) to predict the corresponding liquid-phase TEM images. Figure 1 shows this workflow using the model system of poly( $N$-isopropylacrylamide) (pNIPAM) hydrogel particles. Preliminary MD simulations were performed on PNIPAM particles with varying size and equilibration temperature, based on which liquid-phase TEM images were simulated. These hypothetical, simulated liquid-phase TEM images could be compared with experimental ones, to evaluate consistencies - in parameters such as the shape contour and inner contrast variation - or inconsistencies, which can provide insight into the roles of the electron beam or otherwise non-equilibrium behavior. Potentially, iterative match of the intensity profiles between simulated and experimental liquid-phase TEM images can allow one to infer molecular-level details out of liquid-phase TEM movies/images bearing nanoscale morphology information [5]. 

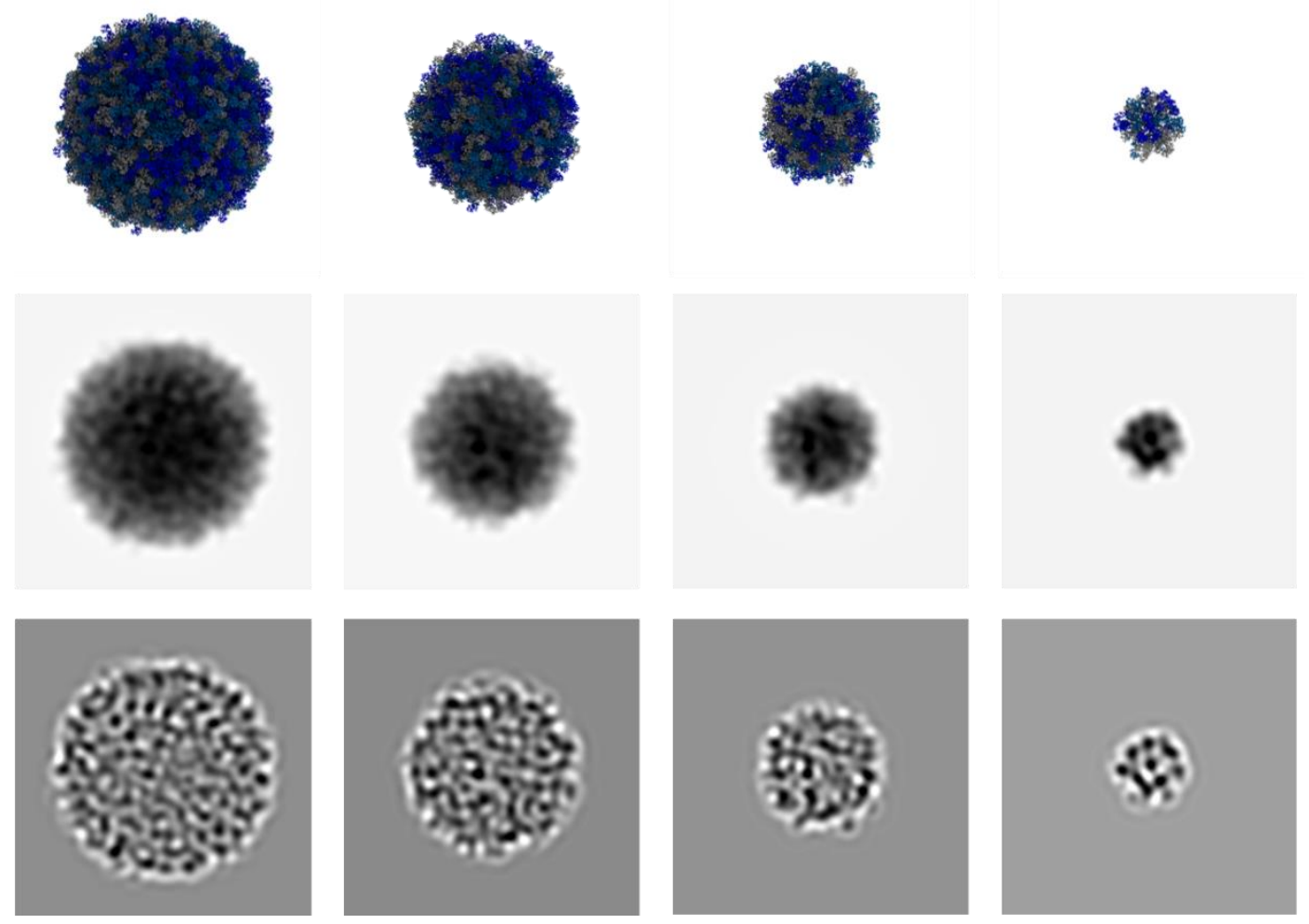

Figure 1. (top) Snapshots from MD simulations of pNIPAM hydrogel particles and corresponding simulated liquid-phase TEM images assuming either (middle) in-focus imaging and an incoherent beam, due to a thick liquid environment, or (bottom) a defocus of $-512 \mathrm{~nm}$ and an incoherent beam (top). From left to right, the particle size decreases from $100 \mathrm{~nm}$ to $75 \mathrm{~nm}, 50 \mathrm{~nm}$, and $25 \mathrm{~nm}$.

\section{References}

[1] H Wu, et al., Adv. Mater. 32 (2020), 2001582.

[2] A. Ianiroet al.,Nat. Chem.11 (2019), p. 320.

[3] A. C. Varanoet al., Chem. Commun.51 (2015), p. 16176.

[4] S. Keskin and N. de Jonge, Nano Lett.18 (2018), p. 7435.

[5] The authors acknowledge funding from Air Force Office of Scientific Research (AFOSR project FA9550-20-1-0257). 